\title{
RAGUSA TRA GLI ARAGONESI DI NAPOLI E I TURCHI
}

\author{
Momčilo Spremić
}

Dopo aver conquistato Napoli, il re Alfonso adottò una politica attivissima nei confronti dell'Oriente. Egli lo fece quale erede degli Angiò di Napoli e quale erede dei conquistatori catalani che avevano occupato alcune parti dell'Impero bizantino già nel secolo xIv. Quindi gli stava assai a cuore il titolo di duca di Atene e di Neopatra. Egli manteneva rapporti con tutti i regnanti di certo prestigio dell'Asia anteriore, con quelli dell'Africa settentrionale e con quelli della Penisola balcanica'. Considerato che i Turchi in quell'epoca stavano creando in Oriente un impero, i cui confini occidentali sboccavano sulla costa orientale dell'Adriatico, è del tutto comprensibile perché quella seguita dal re Alfonso verso i Turchi occupasse un posto centrale nel quadro generale della sua politica orientale. Del resto, indipendentemente dal suo atteggiamento personale, egli dovette adottare

${ }^{1}$ Sulla politica orientale del re Alfonso esiste un lavoro piuttosto vasto sebbene assai invecchiato, ancor sempre prezioso però per i molti documenti integri pubblicativi: F. CERONE, La politica orientale di Alfonso D'Aragona, Archivio Storico per le province Napoletane, anno 27/1902/3-93; 380-456; 555-634; 774-852; anno 28/1903/154-212. Sunti del lavoro di F. Cerone sono stati pubblicati da J. MiRET y SANS, La politica oriental de Alfonso V de Aragón, Barcelona 1904. 
una politica specifica nei confronti dei Turchi, essendo egli re d'Italia cristiano.

Nella politica seguita dal re Alfonso nei confronti dei Turchi, Ragusa occupò un posto di particolare interesse. Valendosi di una collocazione geografica favorevole, con dei sudditi che andavano commerciando in un'area che si stendeva da Costantinopoli, dalla Siria e da Alessandria d'Egitto, attraverso le terre balcanice, fino a Napoli, a Siracusa e a Palermo, Ragusa poteva prestare grandi servizî all'Aragonese. D'altra parte anche la Repubblica contava di approfittare del re Alfonso per la lotta contro i Turchi.

Quando tra il 1443 e il 1444 venne promossa una grand'azione dei crociati, il papa Eugenio IV, incitando i Ragusei a prender parte alla guerra, rilevava che in quell'impresa avrebbero partecipato, oltre alle altre navi, anche quelle inviate dal re aragonese. Infatti, la Repubblica seguì con grande interesse il concentramento della flotta crociata di re Alfonso a Trani, essa scambiò messaggi col re di Napoli e vi inviò anch'essa due galee equipaggiate da circa 430 uomini. Naturalmente, e il re Alfonso e i Ragusei desideravano vedere i Turchi cacciati dall'Europa, ma ambedue le parti badavano ai proprî interessi. Ancora prima dell'inizio dell'impresa, Alfonso aveva chiesto al re ungherese Ladislao di fargli avere Atene e Neopatra appena che fossero state liberate dai Turchi, mentre la Repubblica di Ragusa contava di ottenere Valona e Canina al momento della spartizione della Turchia europea².

Dopo la sconfitta presso Varna Giovanni Hunyadi aveva in mente di continuo di vendicarsi contro i Turchi. Egli si preparò

2 B. KREKIC, Dubrovnik /Raguse/ et le Levant au Moyen age, Paris-La Haye 1961, 56, 334-335/num. 1027/, 336 /num. 1036/, 340-341/num. 1061/; J. RADONIĆ, Acta et diplomata Ragusina, I, Belgradi 1934, 467-468, 481-482; N. JORGA, Notes et extraits pour servir à l'histoire des croisades au Moyen age, II, Paris 1899, 406, 411-412. Cf. B. KREKIC, Učeśce Dubrovčana u ratovima protiv Turaka 1443. i I444. g./La partecipazione dei Ragusei alle guerre contro i Turchi negli anni 1443 e 1444/, Zbornik radova Vizantoloskog instituta, 2, Beograd 1953, 146-152. Sui piani del re Alfonso di promuovere la crociata: S.S. VIDAL, "Sobre el ideal de cruzada en Alfonso V de Aragón", Hispania, t. XII, num. 47, Madrid 1952, 232-252. 
alla guerra che sarebbe finita con la rotta degli Ungheresi a Cossovo nel $1448^{\text {? }}$. I Ragusei informavano regolarmente il re di Napoli sui preparativi di guerra sia quelli svolti dal governatore ungherese che su quelli effettuati dal sultano. Essi gli diedero notizie sul movimento di tutti e due gli eserciti come pure sulla stessa battaglia di Cossovo ${ }^{4}$.

Dal 1451 pareva che il re Alfonso avrebbe capitanato la lotta contro i Turchi. In quest'anno egli stipulò l'alleanza col despota di Morea Demetrio Paleologo, conforme alla quale, nel caso dell'espulsione dei Turchi dall'Europa, la corona bizantina sarebbe dovuta passare in possesso dell'ambizioso re di Napoli. Tuttavia, anche se lo stesso imperatore bizantino Costantino XI, chiedendo aiuto, offriva nel 1452 l'isola Lemno al re Alfonso, questi, disponendo di una flotta piuttosto scarsa, occupato sempre nella politica italiana, non potè intraprendere un'azione efficace contro i Turchi. In verità egli regalò una certa quantità di grano all'imperatore bizantino e mandò anche, insieme al papa e a Venezia, alcune galee a difendere la Costantinopoli assediata. Questa flotta, però, giunse solo fino a Negroponte al momento dell'entrata di Maometto II nella capitale bizantina ${ }^{5}$. Già alla fine del giugno del $1453 \mathrm{i}$ Ragusei informavano re Alfonso sulla caduta di Costantinopoli ${ }^{6}$.

La conquista della «Roma orientale» da parte dei Turchi commosse profondamente il re di Napoli e tutta Italia. Questo avvenimento, tra l'altro suggeri la stipulazione della pace di Lodi del 1454 tra gli stati italiani ratificata dal re di Napoli nel 1455. Dopo

${ }^{3}$ Archivio di Stato di Ragusa. Acta Consilii Rogatorum/Cons, Rog./ XI, 7,7 :

${ }^{4}$ Era stato progettato che il re Alfonso aiutasse con del denaro e coll'esercito l'impresa di Giovanni Hunyadi contro i Turchi e quindi ottenesse in cambio la corona del regno ungherese. L. THALloczy-S. BARABÁs, Codex diplomaticus comitum de Frangepanibus, Budapest 1910, 350-356.

${ }^{5}$ L. VON PASTOR, Geschichte der Päpste seit Ausgang des Mittelalters I, Freiburg im Breisgau 1925, 612-613; C. MARINESCO, «Contribution à l'histoire des relations économiques entre l'Empire byzantin, la Sicile et le royaume de Naples de 1419 à 1453n, Atti del V Congresso internazionale di studi bizantini I, Roma 1939, 218-219.

${ }^{6}$ Cons. Rog. XIII, 207, 231', 236. 
la caduta di Costantinopoli egli chiese ai Ragusei di informarlo regolarmente su tutto ciò che succedesse in Turchia. Nel 1454 egli decise di prender parte di persona alle lotte contro gli Ottomani e quindi nella primavera dello stesso anno inviò attraverso Ragusa un suo ambasciatore diretto ad andar a trovare tutti i signori della Penisola balcanica con cui il re manteneva relazioni d'amicizia'. Nel 1454 la Repubblica informava re Alfonso sulla situazione in Serbia e sui preparativi in corso in Ungheria per una guerra contro i Turchi. L'anno successivo, nel 1455, gli diede anche notizie sull'occupazione turca di Novo Brdo e lo informò sulla dieta di Francoforte come pure sulle conclusioni della dieta tenutasi a Buda nel febbraio dello stesso anno, alla quale $\mathrm{i}$ baroni ungheresi presero impegno di partecipare personalmente alla guerra contro i Turchi ${ }^{8}$.

I Ragusei, naturalmente, informarono il re Alfonso anche sulla sconfitta subita dai Turchi presso Belgrado nel 1456. La vittoria riportata dall'esercito di Hunyadi, nelle cui file militava anche l'abruzzese Giovanni Capistrano, il fautore entusiasta delle crociate, venne celebrata nell'Italia meridionale e in Catalogna. Verso la fine della sua vita il re Alfonso si interessò sempre più dei Turchi nel cui esercito si potevano già sentire gridi di guerra che aizzavano a una spedizione diretta contro Roma e contro le Puglie. I Ragusei lo informarono perciò anche all'inizio del 1458 sulla situazione in Turchia e sull'invio di una loro ambasciata alla Porta?.

Così risulta che la Repubblica mandava regolarmente notizie

7. DE THALLOCZY, «Frammenti relativi alla storia dei paesi situati all'Adrian, Archeografo Triestino, III serie, vol. VII, Trieste 1913, 297.

${ }^{8}$ Cons. Rog. XIV, 169, 178, 196; Acta et diplomata Ragusina, II, 579-581; J. GelCiCh-L. Thalloczy, Diplomatarium relationum reipublicae Ragusanae cum regno Hungariae, Budapest 1887, 571-573. Cf. I. BožIĆ, Dubrovnik i Turska u XIV i XY yeku/Ragusa e Ia Turchia nei secoli XIV e XV/, Beograd 1952, 131-132, 134, 137-138.

'Cons. Rog. XV, 31', 37', 189', 198, 227; V. MAKUŠEv, Istorijski spomenici Južnih Slovena i okolnih naroda/Monumenti storici degli Slavi del Sud e dei popoli vicini/ II, Beograd 1882, 88-91. Spinto dal crescente pericolo dei Turchi nel 1456 re Alfonso mandò in Oriente alcune galee facenti parte della flotta crociata. C. MINIERI Riccio, «Alcuni fatti di Alfonso I di Aragona dal 15 
sui Turchi al re Alfonso. Le informazioni si fecero più frequenti nella seconda metà del suo governo e seguirono in tale maniera lo sviluppo del processo del consolidamento degli Ottomani nella Penisola balcanica, i quali cominciarono a rappresentare un pericolo imminente anche per la stessa Italia e soprattutto per la Puglia. Cosi, per mezzo delle informazioni sui Balcani, e in modo particolare per mezzo delle nuove sull'Ungheria, i Ragusei spingevano il re Alfonso alla guerra contro i Turchi, intanto con le notizie provenienti dall'Italia essi incoraggiavano Giovanni $\mathrm{Hu}$ nyadi a persistere nella lotta contro gli Ottomani. Inoltre, secondo il continuatore della Cronaca Ragusea di Giunio de Resti, nel 1455 la Repubblica avrebbe rassicurato il comandante di Skopje Issa-bei che i regnanti cristiani, in particolare il re aragonese e il papa, fossero avversari dei Ragusei, aggiungendo che i loro sudditi venissero persino maltrattati dal re aragonese il quale avrebbe vietato loro, secondo la stessa fonte, l'esportazione di derrate dalle Puglie ${ }^{10}$.

Mentre il re Alfonso potè fare progetti di come cacciare i Turchi dall'Europa e quindi di salire sul trono di Costantinopoli, suo figlio Ferrante dovette difendere il proprio stato dagli attacchi degli Ottomani. Temendo i Turchi, il re Ferrante drizzava il suo sguardo ai Baicani e quindi anche a Ragusa. Ma anche la Repubblica era premuta dalla minaccia turca e badava bene di non cadere in disgrazia del sultano per ragione qualsiasi. Di conseguenza, alla fine del 1461 il Senato non era disposto a inviare a Skanderberg in Puglia le informazioni sapute da Paladino de Gondola alla Porta".

Anche se il papa Pio II aveva fatto degli sforzi per indire una crociata, ed aveva intenzione di passare per Ragusa e unirsi egli stesso all'impresa, la Repubblica sapeva bene che gli stati italiani non potevano mettersi d'accordo sulla somma, sul numero

aprile 1437 al 31 maggio 1458», Archivio Storico per le province Napoletane $6 / 1881 / 451,453$. Cf. F. BABINGER, Mahmed der Eroberer und seine Zeit, München $1959,159$.

${ }^{10}$ Chronica Ragusina Junii Restii item Joannis Gundulae, Zagrabiae 1893, ed. Sp. Nodilo. 348-349.

i1 I. Božic, op. cit., 340-341. 
delle navi né su quello dei soldati necessarî per la lotta contro i Turchi e quindi cercava di organizzare il meglio possibile la propria difesa della città. Nonostante ciò essa trovò aiuto nell'Italia meridionale al momento quando fu direttamente minacciata dagli Ottomani nel 1463. Nel Principato di Taranto vennero assoldati degli uomini capitanati da un certo Spirito di Altamura vicino a $\mathrm{Bari}^{12}$. In verità il re Ferrante, dopo essersi assicurato il trono, cominciò ad aiutare indirettamente la difesa di Ragusa contro i Turchi. Esaudendo la domanda del Senato, egli permise alla Repubblica di acquistare nel suo stato del salnitro, materiale di guerra importantissimo. In quest'occasione egli rilevò che lo faceva per la minaccia che premeva Ragusa da parte dei Turchi un popolo di selvagi e infedeli.

Intanto, col passar del tempo i Ragusei cominciarono a regolarizzare i loro rapporti coi Turchi e divennero quindi persino un punto di incontro tra Ottomani e Italia. Nel 1466 il Senato di Ragusa cercava di acquistare in Italia dei libri di medicina necessarî al medico del sultano, certo Giacomo di Gaeta, e nel 1470 acconsentiva a un proprio suddito di andare in qualità di ambasciatore di Issa-bei dal re Ferrante, pur avendo vietato un anno prima a Nicola de Palma di assumere una tale missione ${ }^{13}$. Del resto, dagli anni settanta del secolo $\mathrm{xv}$ in poi attraverso Ragusa venne esportata la seta proveniente dalla Turchia nell'Italia meridionale e quindi a Firenze ${ }^{14}$. Perciò durante la guerra condotta tra il 1463 e il 1479 i Veneziani accusavano i Ragusei presso il loro signore supremo, il re Mattia Corvino, di aiutare i Turchi, nemici della Serenissima e di tutta la cristianità ${ }^{15}$. I Ragusei, intanto, ebbero occasione di vedere, proprio durante questa guerra, come re Ferrante mantenesse rapporti col sultano Maometto II. Infatti il re di Napoli mandò nel 1467 alla Porta il suo ambasciatore Bernardo Lopez, invero in seguito a una

12 Acta et diplomata Ragusina II, 648; Annali di Ragusa del Magnifico ms. Nicolo di Ragnina, Zagrabiae 1883, ed. Sp. Nodilo, 261.

${ }^{13}$ Cons. Rog. XIX, 47'

14 Acta Minoris Consilii/Cons. Minus/ XIX, 260; Cons. Rog. XXVII, 81.

15 J. NAGY-A. NyȦRY, Magyar diplomacziai emlékek Mátyás kiràly koràbol, 1458-1490 II, Budapest 1878, 19-20 /num. 10/. 
richiesta inviatagli dal sultano. Pare che, in quell'occasione, Maometto II, oltre a regolare i rapporti commerciali tra Turchia e Regno di Napoli, avesse voluto attirare a sé il re Ferrante per la lotta contro i Veneziani ${ }^{16}$. Perciò in Italia corse voce caldeggiata in particolare dai Veneziani che il re di Napoli e il sultano avessero concluso un contratto. A Ragusa sapevano che ciò non era vero e quindi è di lì che giungevano in Italia e soprattutto nell'Italia settentrionale notizie sulla situazione reale. Invero, sebbene il sultano avesse scritto a Ferrante anche nel 1470, informandolo sull'occupazione del Negroponte veneziana, e sebbene gli si fosse rivolto anche più tardi, il re di Napoli non accettò mai la mano portagli da Maometto II $^{17}$. Pur avendo i Veneziani accusato Ferrante di permettere alle navi turche di servirsi dei suoi porti per la lotta contro Venezia, il re di Napoli non solo respingeva tali accuse come non vere, ma nel 1472 egli inviò in Oriente alcune galee a far parte della flotta crociata. Egli si sforzava, insieme ad altri regnanti cristiani, di cattivarsi l'amicizia di un avversario del sultano, precisamente del signore di Caramania Uzun-Hassan, e nel 1474 aiutò la guarnigione della Scutari veneziana assediata fornendole del grano $^{18}$.

Pertanto, appena che Venezia ebbe concluso la pace col sultano /1479/, Ferrante fu minacciato direttamente dal pericolo dei Turchi. In Albania si era radunato un forte esercito turco che, come pensava al principio lo stesso re di Napoli, sarebbe andato contro Ragusa. Però, il 28 luglio del 1480, quest'esercito sbarcò nei pressi di Otranto e l'11 agosto occupò la città. Circa 1000 sudditi del re Ferrante persero la vita, tra loro anche Francesco Zurlo, il comandante della difesa e anche l'arcivescovo di Otranto

${ }^{16}$ F. TRINCHERA, Codice aragonese o sia lettere regie, ordinamenti ed altri atti governativi de' sovrani aragonesi in Napoli riguardanti l'amministrazione interna del reame e le relazioni all'estero I, Napoli 1866, 87-88 /num. 65/, 98 101 /num. 70/, 102 /num. 71/.

${ }^{17}$ Notes et extraits, IV, 286-287. Alla corte di Napoli soggiornava nel 1470 "Citerby de Servia», ambasciatore del sultano Maometto II. N. BARONE, «Le cedole di Tesoreria dell'Archivio di Stato di Napoli, dall'anno 1480 al 1504», Archivio Storico per le province Napoletane, 9, /1884/, 230.

${ }^{18}$ M. Drinov, Socinenija Opere, I, Sofija 1909, 87. 
Stefano Pendinelli, un vecchio di 80 'anni. 8000 persone circa furono condotte schiave in Albania, mentre 800 abitanti di Otranto vennero incatenati e trascinati su un monte vicino e ivi trucidati perché non volevano accettare la religione musulmana. I loro corpi furono lasciati come cibo ai cani randagi e ai rapaci ${ }^{19}$.

La Repubblica di Ragusa non si ingeri nella crisi di Otranto, seguiva però con grande attenzione lo sviluppo degli avvenimenti ${ }^{20}$. Le sue simpatie, naturalmente, erano dalla parte cristiana, ma per timore dei Turchi, non osava venire in aiuto al re Ferrante. La paura dei Turchi era intanto maggiore in quanto il sultano aveva chiesto, prima di sbarcare a Otranto, a Ragusa di consegnargli tutti i possedimenti fuori delle mura urbane. Premuta dalla gran paura, la Repubblica mandò ambasciatori a Napoli, a Roma e in Ungheria chiedendo aiuto e consiglio. Alla vigilia dello sbarco a Otranto il governo di Ragusa richiamò i proprî sudditi dalle Puglie, dalle Marche e permise agli abitanti di Lagosta di rifuggiarsi dovunque volessero e sapessero, nelle Puglie, in Dalmazia, a Venezia, a Ragusa o in qualsiasi altro luogo. Ciò nonostante i sudditi di Ragusa aiutarono il re Ferrante, ufficialmente all'insaputa della Repubblica, entrando al suo servizio. Fatto sta che per Otranto combatterono come fanti dalla parte del re Ferrante molti mercenari di Ragusa, tra i quali si distinsero in modo particolare Pietro e Pasquale de Biagio di Ragusa. Inoltre, nel luglio del 1418, alla vigilia della capitolazione della guarnigione turca di Otranto, a Ragusa soggiornò l'ambasciatore del re Ferrante, ma questa missione fu tenuta in segreto dalla Repubblica. Essa però non nascose la propria gioia per la riconquista di Otranto da parte del re Ferrante e quindi

${ }^{19}$ Sulla crisi di Otranto esiste una immensa letteratura. Mentre alcuni auto$\mathrm{ri}$, in particolare quelli piuttosto vecchi, tendevano a dimostrare come Venezia non avesse avuto alcuna parte nella conquista turca di Otranto, gli altri accusavano la Serenissima di aver operato in concerto con gli Ottomani in occasione del loro sbarco in Puglia. F. FossaTr, Sulle cause dell'invasione turca in Italia nel 1480, Vigevano 1901.

${ }^{20}$ I cronisti ragusei hanno descritto minutamente gli avvenimenti riguardanti la conquista turca di Otranto. Annales ragusini anonymi, Zagrabiae 1883, ed. Sp. Nodilo, 71-72; Copioso ristretto degli Annali di Ragusa di Giacomo di Pietro Luccari, Ragusa 1790, 188-190. 
nel settembre del 1418 l'emissario del re, che aveva portato questa notizia, venne premiato con 20 ducati $^{21}$.

Durante la crisi di Otranto la Repubblica mandava informazioni preziose al re di Napoli. Infatti inviava notizie sulla situazione nella Penisola balcanica al re Ferrante nello stesso modo come lo aveva fatto al tempo di Alfonso. Solo che durante il governo di Ferrante, essendo stato il Regno di Napoli direttamente minacciato dai Turchi, al re interessava ogni notizia su di loro e quindi egli fece diventare Ragusa il suo più importante centro di informazioni sulla costa adríatica orientale. Mentre che il re Alfonso veniva informato dalle autorità ragusee, a Ferrante fornivano notizie inoltre alcuni suoi informatori tra cui $\mathrm{i}$ più importanti furono Michele de Resti, Paladino de Gondola e Giovanni Givolino. Tutti e tre conoscevano benissimo la situazione nell'Italia meridionale essendo esperti nel commercio proprio con quelle terre. Come ambasciatori di Ragusa essi frequentavano la corte di Napoli e col passar del tempo divennero persone di fiducia del re Ferrante. Inviando da Ragusa delle preziose informazioni, essi aiutavano il re Ferrante, però in cambio ottenevano facilmente da lui molti privilegi per la città nativa, rendendole in tal modo grandi favori. Naturalmente, le informazioni ricevute da Ragusa non furono utili solo al re Ferrante. Dai documenti pubblicati da F. Trinchera risulta che il re di Napoli faceva diramare le notizie ricevute da Ragusa in tutta Italia ${ }^{22}$.

Pur incitando con le loro notizie il re Ferrante alla guerra contro i Turchi, i Ragusei nel contempo continuarono a regolarizzare i proprî rapporti col sultano. I Ragusei tenevano bene informato il re di Napoli sulla situazione in Turchia, mentre tacevano gli affari svolti dalla Repubblica alla Porta. Per esempio, nel 1484, mentre aveva inviato alla corte del sultano i suoi ambasciatori

21 E'noto che nel febbrario del 1481 il re Ferrante aveva assunto al proprio servizio i seguenti Ragusei: «Pascal de Biasi de Ragusa capo de dicti fanti, Gerabiso de Ragusa, Falich, Rado Buscasino vechio, Rado Prinatovich, Vlatico Univich, Alegreto de Branovich, Malo Rado Buretich, Brischio de Brulovich, Rado Popovich etc.». J. NaGY-A. NYẢRY, op. cit., III, 5 /num. 4/.

${ }^{22}$ F. TRINCHERA, op. cit., I, 128-129/num. 94/, $130 /$ mum. 95/, 1I/1, 96 /num. 113/, 102-103 /num. 122/, 124-127 /num. 143/. 
affinché risolvessero la questione della posizione dei proprî sudditi in Turchia, Ragusa informò Napoli che il principale compito di questi emissari fosse quello di spiare alla Porta e di inviare delle notizie raccoltevi al re Ferrante ${ }^{23}$. Quando i Turchi minacciavano la loro città, i Ragusei sapevano strombazzando mettere in allarme tutti come se si trattase di un pericolo per l'Italia e per tutta la cristianità. Intanto, tacevano le facilitazioni commerciali ottenute dal sultano come anche la regolarizzazione della posizione dei loro sudditi nell'Imperio ottomano, privilegi di cui godevano grandemente negli anni settanta del secolo XV e non ne informavano l'Occidente, nemmeno il re Ferrante.

Difatti, sebbene avesse continuato a inviare informazioni al re sul movimento dell'esercito turco e sebbene avesse mandato nel 1492 dei falchi come regalo al figlio di Ferrante Federigo, il quale combatteva come capitano della flotta contro i Turchi, cominciando intorno all'anno 1484, Ragusa faceva sempre più frequente da punto di collaborazione tra l'Impero ottomano e l'Italia $^{24}$. In conseguenza, già negli anni ottanta del secolo $\mathrm{xv}$ vennero per mezzo di Ragusa ricomprati dalla prigionia turca dei sudditi del Regno di Napoli ${ }^{25}$. Oltre a ciò, la Repubblica desiderava sempre meno ingerirsi negli urti tra Catalani e Turchi avvenuti vicino alle sue coste ${ }^{26}$. Cosi, per esempio, nel 1490 venne rilasciato il salvacondotto a un certo Paolo de Campo di

23 V. MAKUŠEV, op. cit., II, 55-56. Nel 1482 il re Ferrante stesso scambiava l'ambasciatore col sultano Bayazid II. F. BABINGER, «Sechs unbekannte aragonische Sendschreiben im Grossherrlichen Seraj zu Stambul», Studi in onore di Riccardo Filangieri, II, Napoli 1959, 123. D'altronde, i Ragusei informavano nel secolo XVI i reggenti spagnoli a Napoli sulla situazione in Turchia. J. TADIĆ, Spanija i Dubrovnik u XVI veku, /La Spagna e la Turchia nel secolo $X V I /$, Beograd 1932, 145-146.

${ }^{24}$ Cons. Rog., XXVI, 257; Cons. Minus., XXIV, 195. II re Ferrante riceveva informazioni anche da altre città della costa adriatica orientale. St. NovAKOvić, Italijanski archivi i naśa istorija /Gli archivi italiani e la nostra siorial, Glasnik Srpskog učenog društva, Beograd 1870, 38.

${ }^{25}$ Cons, Rog. XXIV, 252; Diversa Cancellariae, LXXXIV, 99: M. DINIC, Iz Dubrovackog arhiva /Materiali dell'Archivio di Ragusa/, III, Beograd 1967. 169-170 /num. 336/.

26 Cons, Rog. XXIV, 251'; XXVI, 70. 
Catania accompagnato però dall'avvertenza che il lasciapassare non si riferiva ai danni da lui arrecati ai Turchi ${ }^{27}$. Il governo di Ragusa inviava sempre più frequente ai vicini comandanti turchi diversi specialisti, di solito medici, che, d'altronde erano sudditi aragonesi ${ }^{28}$. Poi esso informava anche il medesimo re Ferrante sul commercio esistente tra Turchia e Firenze svolto attraverso l'Italia meridionale da sudditi ragusei ${ }^{29}$.

Servendosi del vecchio e provato espediente, chiedendo al re Alfonso II il permesso di esportare il grano dall'Italia meridionale, nel 1494 i Ragusei rilevavano che la loro città, rimanendo senza alimenti, poteva facilmente diventare preda dei Turchi ${ }^{30}$. Istruiti dall'esperienza i ragusei non davano retta neanche ai progetti del re francese Carlo viI, il quale, dopo aver conquistato Napoli, dichiarava che avrebbe fatto guerra contro i Turchi. Del resto, la Repubblica aveva già regolarizzato i proprî rapporti con la Porta e quindi i mercanti ragusei, muniti di privilegi del sultano, si erano sparsi di nuovo per la Penisola balcanica come ai tempi degli stati cristiani indipendenti.

27 Cons. Rog. XXVI, 117.

28 Cons. Rog. XXV, 247; Cons. Minus, XXIII, 205.

${ }^{29}$ Cons. Minus, XXIV, 39; Acta et diplomata Ragusina, II, 791. Cf. I. Božić, op. cit., 298-299. Un certo tempo, verso la fin del suo governo il re Ferrante ospitò Gem, pretendente al trono turco. V. MAKUŚEV, Italijanski arhivi i hrnajaściesja v nih materiali dlja Slavjanskoj istorii. II Neapolj i Palermo IGli archivi italiani e materiali per la Storia slava serbativi. II Napoli e Paler. mo/, Zapiski imperatorskoj akademii nauk, XIX, prilaženie num. 3, Sankpeterburg 1871, 15-16, Cf. F. BABINGER, Sechs. 124-125.

${ }^{30}$ Acta et diplomata Ragusina, II, 802. 